https://doi.org/10.15407/ujpe65.4.310

V. STASHCHUK, ${ }^{1}$ V. STUKALENKO, ${ }^{1}$ S. ROZOUVAN,${ }^{1}$ V. LYSIUK $^{2}$

${ }^{1}$ Taras Shevchenko National University of Kyiv, Faculty of Physics

(4, Glushkov Ave., Kyiv 03022, Ukraine)

${ }^{2}$ V. Lashkaryov Institute of Semiconductor Physics of the NAS of Ukraine

(41, Nauky Ave., Kyiv 03028, Ukraine)

\title{
METHODS OF CALCULATION \\ OF THE MAGNETO-OPTICAL CHARACTERISTICS OF FERROMAGNETIC MATERIALS ${ }^{1}$
}

\begin{abstract}
We have calculated the optical and magneto-optical properties of ferromagnetics in a wide spectral range $h \nu=1.24-5.15 \mathrm{eV}$, by applying theoretical models which were reported earlier in the literature. This allowed us to find a connection between the conductivity and the measured values of the complex Kerr angle $\tilde{\Theta}$ in order to establish the most proper technique for processing the experimental data. The behavior of the dispersion curves of diagonal and non-diagonal components of the tensor of optical conductivity depends on the choice of signs of the imaginary parts of a complex Kerr angle and a refractive index, which should be taken into account in the data analysis. The differences between dispersion dependences are insignificant in the region of energies higher than $4.5 \mathrm{eV}$. In this paper, the classical theory of free electrons in metals has been applied to give more details about the magneto-optical Kerr effect in ferromagnetic samples.
\end{abstract}

Keywords: magneto-optical properties, optical conductivity tensor, off-diagonal components, Kerr effect, ferromagnetic materials.

\section{Introduction}

From comprehensive perspectives, it is important to consider a variety of approaches studying such complex systems as nanostructured ferromagnetic materials which are embedded in a dielectric matrix. Such objects (structures) are of interest not only from the theoretical, but also from the practical point of view and have already been used for the information recording. It is worth to mention amorphous transition metals with amorphizer embedded in a dielectric matrix as nanogranules. The most commonly used dielectric materials for such purposes are oxides of nontransition elements such as Si and Al. Optical spectral techniques were chosen to effectively look into the electronic structure of such complex objects and their properties, in particular, magneto-optical ones, in order to allow the separation of components of the absorption spectra which were originated from electrons with different spin directions (forward $\uparrow$ and backward $\downarrow$ to the direction of a spontaneous magne-

(C) V. STASHCHUK, V. STUKALENKO, S. ROZOUVAN, V. LYSIUK, 2020 tization). Structures $\left(\mathrm{Co}_{41} \mathrm{Fe}_{39} \mathrm{~B}_{20}\right)_{x}\left(\mathrm{SiO}_{2}\right)_{1-x}$ were selected as objects of the study.

It is especially important to study the optical properties of a continuous amorphous $\mathrm{Co}_{41} \mathrm{Fe}_{39} \mathrm{~B}_{20}$ ferromagnetic matrix on the basis of the measured spectra of real and imaginary parts of the Kerr complex angle over a wide spectral interval. However, the processing of the experimental data meets significant difficulties due to divergences in plus and minus signs of the complex Kerr angle $\tilde{\Theta}_{\mathrm{K}}$ and the complex refractive index $\tilde{n}$ in equations. The final results can differ drastically depending on a choice of the signs.

Before examining the model of electronic structure of $\mathrm{Co}_{41} \mathrm{Fe}_{39} \mathrm{~B}_{20}$ ferromagnetic alloys based on the study of the results of optical and magneto-optical experiments over a wide spectral interval, it is necessary to select the model which should be used in processing the data of magneto-optical experiments in order to describe these properties most properly.

1 The paper was presented at XXIVth Galyna Puchkovska International School-Seminar "Spectroscopy of Molecules and Crystals" (August 25-30, 2019, Odesa, Ukraine).

ISSN 2071-0194. Ukr. J. Phys. 2020. Vol. 65, No. 4 
The Kerr effect was discovered [1] as a light polarization plane rotation after the reflection from a polished surface of ferromagnetic iron. The effect is more often described as a phenomena related to the thirdorder nonlinear susceptibility tensor which is responsible for the induced birefringence in studied samples. Different cases of polar and nonpolar molecules, gases, liquids or solid state media $[2,3]$ may result in different relationships for induced optical nonlinearities. In general, various manifestations of the optical Kerr phenomenon are associated with the birefringence and optical anisotropy in a medium which may have different origin. If we take refraction indices parallel and perpendicular to the anisotropy axis $\left(n_{\|}\right.$, $n_{\perp}$ ), the birefringence which can be induced either by a strong electric field or magnetic macro ordering results in nonequal diagonal components of the dielectric tensor. For the magneto-optical Kerr effect (MOKE) [4], the dielectric tensor has nonzero offdiagonal imaginary components and is not dependent on the light intensity. In the this case, those components are responsible for the light polarization rotation and a light ellipticity variation. For the magnetization perpendicular to the sample surface, the dielectric tensor in the magneto-optical Kerr effect has the following form [5]:

$\varepsilon=\left(\begin{array}{ccc}\varepsilon_{x x} & \varepsilon_{x y} & 0 \\ -\varepsilon_{x y} & \varepsilon_{x x} & 0 \\ 0 & 0 & \varepsilon_{z z}\end{array}\right)$.

This relation was derived, by assuming that

$\varepsilon_{x y}=-\varepsilon_{y x}$.

The real and imaginary parts of the refractive indices of two circular polarized waves $(+$ and - subscript indices) are connected with the tensor components (1):

$\left(n_{ \pm}+i k_{ \pm}\right)^{2}=\varepsilon_{x x} \pm i \varepsilon_{x y}$.

The antisymmetric nonzero off-diagonal components of Eq. (A1) are a result of the symmetry relations for tensors in the presence of a magnetic field $[6,7]$. This is a result of the magnetic ordering in a media as an origin of the optical birefringence. The polarization in media for a propagating light in the magneto-optical Kerr effect can be written as

$P_{i}=\varepsilon_{0}\left(\varepsilon_{i j}-1\right) E_{j} \cos (\omega t)$.
This relation which describes the magneto-optic Kerr effect is a result of the birefringence which is induced by the ferromagnetic ordering. The offdiagonal dielectric tensor components can be evaluated using the classical theory of electrons in atoms under the influence of both electric and magnetic fields of the propagating light:

$\ddot{\mathbf{r}}=-\omega_{0}^{2} \mathbf{r}-\frac{e}{m}(\mathbf{E}+\ddot{\mathbf{r}} \times \mathbf{B})$.

Here, $\mathbf{r}$ describes the electron transverse motion in the $\mathrm{XY}$ plane. Introducing the cyclotron frequency $\omega_{c}=$ $=\frac{e B}{m}$ and plasma frequency $\omega_{p}^{2}=\frac{N e^{2}}{\varepsilon_{0} m}$, we obtain the polarization vectors using solutions of Eq. (A5) for the clockwise and counterclockwise circular polarizations:

$P_{+}=\frac{\varepsilon_{0} \omega_{p}^{2}}{\omega_{0}^{2}-\omega^{2}+\omega_{c} \omega} E_{+}$,
$P_{-}=\frac{\varepsilon_{0} \omega_{p}^{2}}{\omega_{0}^{2}-\omega^{2}-\omega_{c} \omega} E_{-}$.

Comparing Eqs. (3) and (6), we can directly evaluate components of the dielectric tensor $\varepsilon$ :

$\varepsilon_{x x} \approx 1+\frac{\omega_{p}^{2}}{\omega_{0}^{2}-\omega^{2}}$,

$\varepsilon_{x y}=-\varepsilon_{y x} \approx \frac{i \omega_{p}^{2} \omega \omega_{c}}{\left(\omega_{0}^{2}-\omega^{2}\right)^{2}}$.

Taking into account that $\varepsilon_{x x} \gg 1$ from (7), we can estimate the relation between the non-diagonal and diagonal components of the dielectric tensor, which is directly proportional to the magnitude of the magnetic field in a medium in the classical approximation:

$\varepsilon_{x y} / \varepsilon_{x x} \approx \frac{i e B}{m \omega\left(\left(\omega_{0} / \omega\right)^{2}-1\right)}$.

For a visible spectral range outside of the strong absorption bands $\omega \gg \omega_{0}$, we can use (8) to estimate roughly the relation between the values of the diagonal and non-diagonal components of the dielectric tensor with regard for the magnitude of the magnetic field $B$ associated with a magnetization of 1 Tesla: $\varepsilon_{x y} / \varepsilon_{x x} \approx e B /(m \omega) \approx 0.0003$.

Spectral ellipsometry techniques have been frequently used to explore the electronic structure of highly absorptive ferromagnetic materials. It is important to obtain the dispersive curves of materials in a broad spectral interval, starting from vacuum ultraviolet wavelengths and ending at the wavelengths 
corresponding to the intraband transitions of electrons (in most cases, it is in near-infrared spectral region). The most informative dispersive curves from this point of view are the optical conductivity spectral dependences [8]

$\sigma_{x x 1}(h \nu)=4 \pi \varepsilon_{0} n(h \nu) \kappa(h \nu)$,

where $h$ is Planck's constant, $\nu$ is the frequency of light, $\varepsilon_{0}$ is the vacuum permittivity, $n(h \nu)$ and $\kappa(h \nu)$ are the real and imaginary parts of the complex refractive index $\tilde{n}(h \nu)$; since this value is proportional to the interband density of electron states [9]. However, for the ferromagnetic materials, the contributions to the optical conductivity $\sigma_{x x 1}(h \nu)$ can be represented as an additive sum of the conductivities originated from interband transitions of electrons having different spin directions which cannot be taken into account separately in the final ratio. By studying additionally the dispersive curves for the complex Kerr angle $\tilde{\Theta}(h \nu)$ in a certain geometry, it is possible to find out the intrinsic nature of absorption bands, based on the spectral behavior of diagonal components of the optical conductivity tensor

$\tilde{\sigma}_{x x}(h \nu)=\tilde{\sigma}_{y y}(h \nu)=\tilde{\sigma}_{z z}(h \nu)$

(in the case of isotropic materials) and non-diagonal components

$\tilde{\sigma}_{x y}(h \nu)=-\tilde{\sigma}_{y x}(h \nu)=\tilde{\sigma}_{\text {mopt }}(h \nu)=$

$=\sigma_{x y 1}(h \nu)+i \sigma_{x y 2}(h \nu)$.

In the case of nonzero off-diagonal component, $\tilde{\sigma}_{\text {mopt }}(h \nu)$ is the difference between the contributions of the electrons with the spins projections along and oppositely the direction of an applied magnetic field to the final optical conductivity. In our opinion, such a method of complex analysis of the electronic structure of ferromagnetic materials is not properly acknowledged in the scientific community. By definition, the optical conductivity tensor $\tilde{\sigma}$ is given by the expression

$\tilde{\sigma}=\left(\begin{array}{ccc}\tilde{\sigma}_{x x} & \tilde{\sigma}_{x y} & 0 \\ -\tilde{\sigma}_{x y} & \tilde{\sigma}_{x x} & 0 \\ 0 & 0 & \tilde{\sigma}_{x x}\end{array}\right)$,

what is typical of isotropic materials [see (10) and (11)] [10].
Magneto-optical properties of the amorphous ferromagnetic material, $\mathrm{Co}_{41} \mathrm{Fe}_{39} \mathrm{~B}_{20}$, which can be used further as a part of $\left(\mathrm{Co}_{41} \mathrm{Fe}_{39} \mathrm{~B}_{20}\right)_{x}\left(\mathrm{SiO}_{2}\right)_{100-x}$ nanocomposites as a more reliable substance for recording information, were studied in [11]. Various models from the literature connect its optical and magneto-optical properties by describing the relationship between the optical conductivity and the measured values of the real $\theta(h \nu)$ (polarization plane rotation) and complex parts $\eta(h \nu)$ (ellipticity) of the complex Kerr angle $\tilde{\Theta}(h \nu)$. It is obvious that the most accurate model should be determined for the processing of experimental data.

In order to determine the optical conductivity tensor components, a method based on measurements of the complex Kerr angle $\tilde{\Theta}(h \nu)$ was applied [1220]. The relations connecting the component of the optical conductivity tensor $\tilde{\sigma}$ (or dielectric permittivity $\tilde{\varepsilon}$ ) with the complex Kerr rotation angle are presented, for example, in [11, 14-19]. However, the authors introduced diverse expressions which link the refractive index $\tilde{n}$ and the Kerr angle $\tilde{\Theta}: \tilde{n}(h \nu)=$ $=n(h \nu) \pm i \kappa(h \nu), \tilde{\Theta}(h \nu)=\theta(h \nu \pm i \eta(h \nu)$. In some cases, different expressions for $\tilde{n}$ are used: $\tilde{n}=n-i \kappa$ $[10,17]$ and $\tilde{n}=n+i \kappa[11,13]$. The similar situation is with the expression for the complex Kerr angle [11, 14-17]. We have found that such differences are noteworthy and may result in different dispersion curves for non-diagonal components of the optical conductivity tensor $\tilde{\sigma}_{\text {mopt }}(h \nu)$. Let us illustrate the results of calculations which are based on different models in detail.

\section{Experimental}

Composite nanostructures, namely, granules of amorphous ferromagnetic alloy $\mathrm{Co}_{41} \mathrm{Fe}_{39} \mathrm{~B}_{20}$ which were embedded into an amorphous dielectric matrix of $\mathrm{SiO}_{2}$, were produced by the ion-beam deposition. The thicknesses of nanostructured layers in the samples reached $1 \mu \mathrm{m}$ with different concentrations of the magnetic phase: $x=1,0.56,0.41,0.33,0.19$. The size of ferromagnetic particles grew from about 2 to $10 \mathrm{~nm}$ with corresponding rise of the magnetic phase concentration. The samples which were studied in our work contained amorphous ferromagnetic layers on their surfaces with thicknesses of about $1 \mu \mathrm{m}$.

Our measurements were carried out in a wide spectral region $\lambda=0.24-1.0 \mu \mathrm{m}(h \nu=1.24-5.05 \mathrm{eV})$. The

ISSN 2071-0194. Ukr. J. Phys. 2020. Vol. 65, No. 4 
optical properties on the nanoscale were investigated by the spectral ellipsometry approach: the ellipsometric angles $\Psi$ and $\Delta$ were measured at different energies of the photons $h \nu$ at one angle of incidence $\varphi$ close to the pseudo-Brewster angle in this spectral region. The low- and high-energy spectral limits of the measurements were predisposed by strong absorption bands due to nanocomposites with a high concentration of granules. The real $\varepsilon_{1}$ and imaginary $\varepsilon_{2}$ parts of diagonal components of the dielectric permittivity tensor $\tilde{\varepsilon}_{x x}=\varepsilon_{1}-i \varepsilon_{2}$ for the model of semiinfinite isotropic medium in contact with air (with refractive index 1) can be determined by the relations $[8,21]$

$$
\begin{aligned}
& \varepsilon_{1}=n^{2}-\kappa^{2}=a^{2}-b^{2}+\sin ^{2} \varphi, \\
& \varepsilon_{2}=2 n \kappa=2 a b,
\end{aligned}
$$

where

$$
\begin{aligned}
& \text { where } \\
& a=\sin \varphi \operatorname{tg} \varphi \frac{1-\operatorname{tg}^{2} \Psi}{1+(\operatorname{tg} \Psi)^{2}+2 \operatorname{tg} \Psi \cos \Delta}, \\
& b=\sin \varphi \operatorname{tg} \varphi \frac{2 \operatorname{tg} \Psi \sin \Delta}{1+(\operatorname{tg} \Psi)^{2}+2 \operatorname{tg} \Psi \cos \Delta},
\end{aligned}
$$

$\varphi$ is the angle of incidence, $\Psi$ is the azimuth of the restored linear polarization, $\Delta$ is the phase difference between the $p$ and $s$ components of the reflected polarized light.

We can determine the diagonal components of the optical conductivity tensor $\tilde{\sigma}_{x x}$ applying formula (9) which is based on relations (12) and (13).

The magneto-optical study of nanocomposites was carried out using the linear magneto-optical Kerr effect in a polar geometry. This effect comprises in rotating the plane of polarization and the appearance of ellipticity of the light reflected from a medium magnetized perpendicularly to the surface.

The magnitude of the applied magnetic field reached $5 \mathrm{kOe}$ in the geometry of the polar Kerr effect. Measurements were carried out at angles of incidence of 10-15 deg. Since the magnetic field generates the anisotropy in a ferromagnetic film on the sample surface, the reflected light polarization alteration is influenced by the magnetic polarization. The parameters of the optical anisotropy can be described by off-diagonal terms of the Jones matrix

$R=\left|\begin{array}{cc}r_{p p} & r_{p s} \\ r_{s p} & r_{s s}\end{array}\right|$

where $p$ and $s$ mark the linear polarizations of light with orientations parallel and perpendicular to the plane of incidence; $r_{p p}$ and $r_{s s}$ are the amplitude coefficients of $p$ - and $s$-components of reflected linearly polarized light.

The off-diagonal amplitude coefficients $r_{p s}$ and $r_{s p}$ reflect a change in the light polarization from the $p(s)$ to $s(p)$ state. They are anisotropic components and represent parts of reflected light with corresponding polarization. The anisotropic reflection coefficients $\left(r_{p s}, r_{s p}\right)$ have a much smaller amplitude than isotropic ones. The complex angles of the magnetooptical (MO) Kerr effect are determined by the relations

$$
\begin{aligned}
& \theta_{s}+i \eta_{s}=r_{p s} / r_{s s} \\
& -\theta_{p}+i \eta_{p}=r_{s p} / r_{p p}
\end{aligned}
$$

where $\theta_{s}\left(\theta_{p}\right)$ is the real Kerr angle of polarization rotation, and $\eta_{s}\left(\eta_{p}\right)$ is the Kerr ellipticity coefficient.

For small angles of incidence $\varphi$ (close to the normal to the surface), $r_{p s}=-r_{s p}$, and $r_{p p}=r_{s s}$. This means that the complex angle of rotation $\tilde{\Theta}_{\mathrm{K}}$ can be written as

$\tilde{\tilde{\Theta}}_{\mathrm{K}}=\theta+i \eta=r_{p s} / r_{s s}$.

\section{Method of Calculations}

Let us begin by considering a model, where the ratio between the complex Kerr angle $\tilde{\Theta}$ and the diagonal and off-diagonal components of the optical conductivity tensor is written as [14]

$\tilde{\Theta}=-\frac{\tilde{\sigma}_{x y}}{\tilde{\sigma}_{x x} \sqrt{1+(4 \pi / \omega) \tilde{\sigma}_{x x}}}$,

where $\tilde{\sigma}_{x x}$ and $\tilde{\sigma}_{x y}$ are complex diagonal and offdiagonal optical conductivity components, respectively, and $\omega$ is the angular frequency.

In another model, the non-diagonal components of the dielectric constant tensor were calculated by the formula [16]

$\tilde{\Theta}=\frac{n_{0} \tilde{\varepsilon}_{x y}}{\sqrt{\tilde{\varepsilon}_{x x}}\left(n_{0}^{2}-\tilde{\varepsilon}_{x x}\right)}$,

where the complex Kerr angle $\tilde{\Theta}, n_{0}$ is the upper medium index of refraction (in our case, $n=1$ for air), the diagonal components of the dielectric permittivity tensor are $\tilde{\varepsilon}_{x x}$, off-diagonal components of the dielectric tensor $\tilde{\varepsilon}_{x y}=\varepsilon_{x y 1}+i \varepsilon_{x y 2}$. The components of the optical conductivity tensor were calculated [17] 


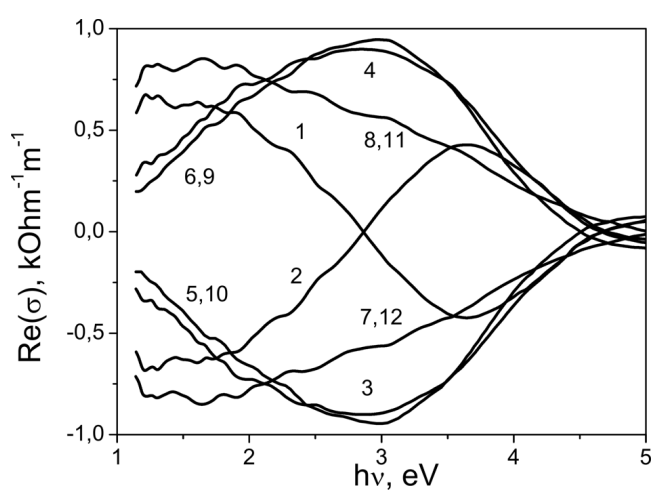

Fig. 1. Spectral dependences of the real $\sigma_{x y 1}$ component of the optical conductivity tensor of amorphous ferromagnetic alloys calculated by expressions (14) - curves 1-4; (15) - curves 5-8; (16) - curves 9-12 by choosing different signs (options A, $\mathrm{B}, \mathrm{C}, \mathrm{D})$ in the expressions for the complex Kerr angle $\tilde{\Theta}(h \nu)$ and refractive index $\tilde{n}(h \nu) \mathrm{A}$ - curves $1,5,9 ; \mathrm{B}$ - curves 2, 6, 10; C-curves 3, 7, 11; D - curves 4, 8, 12

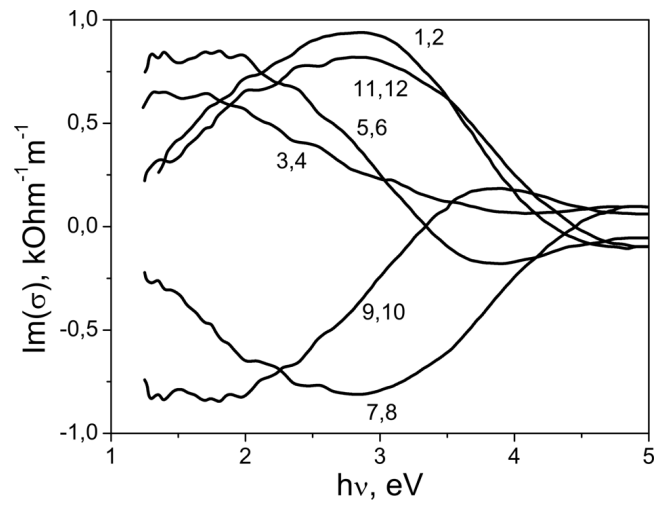

Fig. 2. Spectral dependences of the imaginary parts $\sigma_{x y 2}$ of non-diagonal components of the optical conductivity tensor of amorphous ferromagnetic alloys calculated by expressions (14) - curves 1-4 (dotted lines); (15) - curves 5-8 (dot lines); (16) - curves 9-12 (solid lines) with different signs (A, B, C, D) in the expressions for the complex Kerr angle $\tilde{\Theta}(h \nu)$ and refractive index $\tilde{n}(h \nu)$. A - curves $1,5,9$; B - curves 2, 6, 10; $\mathrm{C}-$ curves $3,7,11 ; \mathrm{D}-$ curves $4,8,12$

applying the formula $\tilde{\varepsilon}_{i j}=\delta_{i j}-i \frac{4 \pi}{\omega} \tilde{\sigma}_{i j}$, where the indices $i, j$ acquire the values $x x$ or $x y, \delta_{i j}=1$ if $i=j$ and $\delta_{i j}=0$, if $i \neq j$.

In [17], the relations for the off-diagonal components of the optical conductivity tensor were expressed in terms of the real and imaginary parts of the complex Kerr angle and the refractive index

$$
\begin{aligned}
\sigma_{x y 1} & =\frac{\omega}{4 \pi}(B \theta+A \eta), \\
\sigma_{x y 2} & =\frac{\omega}{4 \pi}(A \theta+B \eta),
\end{aligned}
$$

\section{4}

where

$A=n^{3}-3 n k^{2}-n$,

$B=-k^{3}+3 n^{2} k-k$.

\section{Results and Discussions}

Let us start to review the calculation results applying different models of off-diagonal components of the optical conductivity tensor $\tilde{\sigma}_{x y}$. We look at the possible options for signs in the expressions for the complex Kerr angle $\tilde{\Theta}(h \nu)$ and the refractive index $\tilde{n}(h \nu)$ :

$A("++"): \tilde{\Theta}(h \nu)=\theta+i \eta, \tilde{n}(h \nu)=n+i \kappa ;$

$B("--"): \tilde{\Theta}(h \nu)=\theta-i \eta, \tilde{n}(h \nu)=n-i \kappa ;$

$C("-+"): \tilde{\Theta}(h \nu)=\theta-i \eta, \tilde{n}(h \nu)=n+i \kappa ;$

$D("+-"): \tilde{\Theta}(h \nu)=\theta+i \eta, \tilde{n}(h \nu)=n-i \kappa$.

The dispersion curves of the real and imaginary parts of off-diagonal components of the optical conductivity tensor $\tilde{\sigma}_{x y}(h \nu)$ which were obtained applying expressions (14)-(16) taken from [14, 16, 17] by choosing different signs (A, B, C, D) in the formulas for the complex Kerr angle and refractive index are shown in Figs. 1 and 2.

As we can see in Fig. 1, some pairs of curves describing the real components of the tensor $\tilde{\sigma}_{x y}(h \nu)$ almost overlap (each curve is marked by two numbers), some curves are identical lat positive $(4,9)$ or negative $(3,10)$ number intervals], others have the same numbers, but opposite signs (its absolute values are the same).

The imaginary parts of non-diagonal components of the tensor $\tilde{\sigma}_{x y}(h \nu)$ dispersion curves are shown in Fig. 2. Numerical calculations were carried out applying expressions: (14)-(16). Pairs of curves calculated applying different signs in expressions (A-D) and which almost overlap are marked by two numbers each. Some curves are identical [in the case of positive numbers for $\sigma_{x y 2}(1,2)$ and $(11,12)$, respectively, A, B versions of expression (14) and C, D versions of expression (16)]; the others have the same values, but opposite signs [absolute values $(7,8)$ and $(11,12)$ of C, D, versions of expressions (15) and (16) respectively].

Performing the comparative analysis of the obtained data and making the simultaneous change of the two signs "++" (case A) to "--" (case B) in the expressions for the complex Kerr angle and the

ISSN 2071-0194. Ukr. J. Phys. 2020. Vol. 65, No. 4 
refractive index, we can formulate three imperative conclusions. First, let us pay attention to those curves (Fig. 1) which have the same absolute values and opposite signs [ 1 and 2; 5 and $6 ; 9$ and 10 calculated by expressions (14), (15), (16), respectively]. Second, other dependences calculated with different expressions are identical, though the calculations were conducted with different pairs of signs (Fig. 1, curves 5 and $11 ; 6$ and 9$)$. Third, the $\sigma_{x y 2}(h \nu)$ dependences (Fig. 2, curves 1 and 2, 5, and 6; 9 and 10) almost coincide, though we used opposite signs during the calculations (A, B expressions).

We should note that the curves $\sigma_{x y 2}(h \nu)$ and $-\sigma_{x y 2}(h \nu)$, which were calculated by expressions $(15)$ and (16), almost overlap.

Let us now find out how a change in the pair of signs in C expression influences D expression. There are cases where $\sigma_{x y 1}$ (with certain values of the energy $h \nu$ ) have approximately the same magnitudes with simultaneously opposite signs [pairs 5 and $7 ; 13$ and 15; 21 and 23 calculated by expressions (14), (15), (16), respectively]; some curves almost overlap, although the different pairs of signs in the expressions were used in their calculations (13 and 23, 15 and 21).

The values of $\sigma_{x y 2}$ obtained using expressions (14)(16) coincide (6 and 8, 14 and 16; 22 and 24, respectively) with $\sigma_{x y 2}$ [expression (15)] and are almost identical with the values $-\sigma_{x y 2}$ calculated by expression (16).

As a result, we have three types of dependences of the real and imaginary parts applying four different options of signs in the $\tilde{n}$ and $\tilde{\Theta}$ expressions in the calculation of non-diagonal components of the optical conductivity tensor $\tilde{\sigma}$. Some curves are similar, some are shifted upward or downward the horizontal axis. Furthermore, the character of the dispersion component of the optical conductivity tensor $\sigma_{x y}(h \nu)$ depends on the choice of signs in the expressions for $\tilde{n}$ and $\tilde{\Theta}$.

As we can see, the choice of different expressions $(\mathrm{A}-\mathrm{D})$ results in different dispersion curves. The question then arises as to what proper theoretical model for the optical conductivity tensor should be chosen.

\section{Conclusions}

Thus, the character of the dispersion dependences of the diagonal and non-diagonal components of the optical conductivity tensor depends on the preference of signs at the both imaginary part of the complex Kerr angle and the refractive index, which should be taken into account in the processing of experimental data. In the region of energies above $4.5 \mathrm{eV}$, the differences between dispersion dependences are insignificant. Consequently, it is necessary to apply a balanced approach to the selection of expressions containing complex values at some point in calculations of magneto-optical parameters of ferromagnetic materials.

\section{APPENDIX A}

The derivation of the relation for the ellipticity of light in the magneto-optical Kerr effect

A classical approximation for quasifree electrons in a metallic ferromagnetic material was applied tp the magneto-optics Kerr effect. A fundamental limitation was shown in the model frames to be responsible for a fundamental limitation on offdiagonal tensor components which is determined by the basic mass-to-charge ratio of the electron.

The tensor of dielectric permeability for a surface with a magnetization perpendicular to the surface reads

$\varepsilon=\left|\begin{array}{lll}\varepsilon_{x x} & \varepsilon_{x y} & 0 \\ -\varepsilon_{x y} & \varepsilon_{x x} & 0 \\ 0 & 0 & \varepsilon_{x x}\end{array}\right|$

The tensor is associated with the refractive indices for clockwise (index " $\mathrm{r}$ ") and counterclockwise (index "l") of circularly polarized light

$n_{r, l}^{2}=\varepsilon_{x x} \pm i \varepsilon_{x y}$.

We can write an expression which connects the intensity of the electric fields of the incident and reflected right and left circularly polarized light waves and its parameters of ellipticity $\frac{E_{r}}{E_{l}}=\frac{\left|E_{r}\right|}{\left|E_{l}\right|} \exp \left(i\left(\alpha_{r}-\alpha_{l}\right)\right)$.

Assuming that $n_{l} \approx n_{r}, \alpha_{r} \approx \alpha_{l},\left|E_{r}\right| /\left|E_{l}\right| \approx 1$ and applying Fresnel's formulas for the normally reflected light, the expression (A3) can be written as

$\frac{1-n_{r}}{1-n_{l}} \frac{1+n_{l}}{1+n_{r}} \approx\left(1+\frac{n_{l}-n_{r}}{1-n_{r} n_{l}}\right)=$

$=\left(\cos \left(\alpha_{r}-\alpha_{l}\right)+i \sin \left(\alpha_{r}-\alpha_{l}\right)\right) \approx\left(1+i\left(\alpha_{r}-\alpha_{l}\right)\right)$.

The phase difference $\alpha_{r}-\alpha_{l}$ for the right and left circularly polarized reflected light is registered as a complex angle $\theta+$ $+i \eta$ which characterizes elliptically polarized light and can be written using expression (A4) in the form

$i(\theta+i \eta)=\frac{n_{r}-n_{l}}{n_{r} n_{l}-1}$

Using expression (A2) and accounting for expression (A5) and $\varepsilon_{x x} \gg \varepsilon_{x y}$, we can write

$\theta+i \eta=i \frac{\sqrt{\varepsilon_{x x}+i \varepsilon_{x y}}-\sqrt{\varepsilon_{x x}-i \varepsilon_{x y}}}{\sqrt{\varepsilon_{x x}+i \varepsilon_{x y}} \sqrt{\varepsilon_{x x}-i \varepsilon_{x y}}-1} \approx$ 


$$
\approx \frac{i \varepsilon_{x y}}{\left(\varepsilon_{x x}-1\right) \sqrt{\varepsilon_{x x}}}
$$

The relation between the diagonal and off-diagonal components of the permittivity tensor in the classical approximation for quasifree electrons in a ferromagnetic material is approximately determined by such basic physical quantity as the mass-tocharge ratio of the electron. For the visible spectral region and for typical ferromagnet magnetization values, the non-diagonal components of the dielectric tensor, which are responsible for the optical activity of a material, are three orders of magnitude smaller than the tensor diagonal components. This fundamental limitation determines the relative weakness of magnetooptical Kerr effect manifestations.

1. John Kerr. On rotation of the plane of polarization by reflection from the pole of a magnet. Phil. Magaz. 3, 321 (1877).

2. A.D. Buckingham. Theoretical studies of the Kerr effect I: Deviations from a linear polarization law. Proc. Phys. Soc. A 68, 905 (1955).

3. A.D. Buckingham. Theoretical studies of the Kerr effect II: The influence of pressure. Proc. Phys. Soc. A 68, 910 (1955).

4. L. Uba, S. Uba, V.N. Antonov. Magneto-optical Kerr spectroscopy of noble metals. Phys. Rev. B 96, 235132 (2017).

5. Xiang Gao, John A. Woollam, R.D. Kirby, D.J. Sellmyer, C.T. Tanaka, J. Nowak, J.S. Moodera. Dielectric tensor for magneto-optic NiMnSb. Phys. Rev. B 59, 9965 (1999).

6. I.M. Boswarva, R.E. Howard, A.B. Lidiard, C.A. Coulson. Faraday effect in semiconductors. Proc. Royal Soc. London A 269, 125 (1962).

7. W.H. Kleiner. Space-time symmetry of transport coefficients. Phys. Rev. 142, 318 (1966).

8. V. Staschuk, V. Kravets, V. Lysiuk, O. Polyanska, V. Stukalenko, A. Yampolsky. Structure and optical properties of $\left(\mathrm{Co}_{41} \mathrm{Fe}_{39} \mathrm{~B}_{20}\right)_{x}\left(\mathrm{SiO}_{2}\right)_{1-x}$ nanocomposites. Ukr. J. Phys. $62(8), 662$ (2017).

9. D.H. Seib, W.E. Spicer. Photoemission and optical studies of Cu-Ni alloys. Phys. Rev. B 2, 1676 (1970).

10. K.H.J. Buschow, P.G. van Engen, R. Jongebreur. Magnetooptical properties of metallic ferromagnetic materials. J. Magn. Magn. Mater. 38, 1 (1983).

11. S. Uba, A. Bonda, L. Uba, L. V. Bekenov, V.N. Antonov, A. Ernst. Electronic structure and magneto-optical Kerr effect spectra of ferromagnetic shape-memory Ni-Mn-Ga alloys: Experiment and density functional theory calculations. Phys. Rev. B 94, 054427 (2016).

12. P.N. Argyres. Theory of the Faraday and Kerr effects in ferromagnetics. Phys. Rev. 97, 2, 334 (1955).

13. M. Born, E. Wolf. Principles of Optics (Pergamon Press, 1991).

14. S. Bordács, I. Kézsmárki, K. Ohgush, Y. Tokura. Experimental band structure of the nearly half-metallic $\mathrm{CuCr}_{2} \mathrm{Se}_{4}$ : An optical and magneto-optical study. New J. Phys. 12, 053039 (2010).
15. P.M. Oppeneer, J. Sticht, T. Maurer, J. Köbler. Ab initio investigation of microscopic enhancement factors in tuning the magneto-optical Kerr effect. Zeitschrift für Physik B Condensed Matter. 88, 309 (1992).

16. Chun Yeol You, Sung Chul Shin. Determination of the offdiagonal element of the dielectric tensor without measuring the ellipticity. Appl. Phys. Lett. 68, 2882 (1996).

17. W. Reim, J. Schoenes. Magneto-optical spectroscopy of felectron systems. In: Handbook of Ferromagnetic Materials, edited by K.H.J. Buschow, E.P. Wohlfarth (NorthHolland, 1990), Ch. 2, pp. $133-236$.

18. S. Patankar, J.P. Hinton, J. Griesmar, J. Orenstein, J.S. Dodge, Xufeng Kou, Lei Pan, Kang L. Wang, A.J. Bestwick, E.J. Fox, D. Goldhaber-Gordon, Jing Wang, and Shou-Cheng Zhang. Resonant magneto-optic Kerr effect in the magnetic topological insulator Cr: $\left(\mathrm{Sb}_{x}, \mathrm{Bi}_{1-x}\right)_{2} \mathrm{Te}_{3}$. Phys. Rev. B 92, 214440 (2015).

19. Frederic J. Kahn, P.S. Pershan, J.P. Remeika. Ultraviolet magneto-optical properties of single-crystal orthoferrites, garnets, and other ferric oxide compounds. Phys. Rev. 186 (3), 891 (1969).

20. Sang Y. Kim, Sang J. Kim, Hun Seo, Myong R. Kim. Complex refractive indices of GeSbTe-alloy thin films: Effect of nitrogen doping and wavelength dependence. Jpn. J. Appl. Phys. 38, 1713 (1999).

21. L.V. Poperenko, Yu.V. Kudrjavtsev, V.S. Stashchuk, Yang Pak Li. Optics of Metal Structures (Kyiv University, 2013) (in Ukrainian).

Received 06.11.19

В. Стащук, В. Стукаленко,

С. Розуван, В. Лисюк

\section{МЕТОДИ РОЗРАХУНКУ}

МАГНІТО-ОПТИЧНИХ ХАРАКТЕРИСТИК

ФЕРОМАГНІТНИХ МАТЕРІАЛІВ

$\mathrm{P}$ е 3 ю м е

В роботі досліджено в широкій спектральній області $h \nu=$ $=1,24-5,15$ еВ оптичні і магніто-оптичні властивості феромагнітних сплавів із застосуванням різних моделей, описаних в літературі. Це дозволило знайти зв'язок провідності з виміряними значеннями комплексного кута Керра $\tilde{\Theta}$ для встановлення найбільш адекватних методів обробки експериментальних даних. Хід дисперсійних залежностей діагональних і недіагональних компонент тензора оптичної провідності залежить від вибору знака перед уявною частиною комплексного кута Керра і показника заломлення, що слід враховувати при обробці відповідних даних. В області енергій більше 4,5 еВ відмінності між дисперсійними залежностями є несуттєвими. У цій роботі застосовується класична теорія вільних електронів металів, щоб дати більш детальну інформацію про магніто-оптичний ефект Керра у феромагнітних зразках.

ISSN 2071-0194. Ukr. J. Phys. 2020. Vol. 65, No. 4 